\title{
Is dark energy a consequence of quantum fluctuations?
}

\author{
Michael Dopita
}

Received: 17 December 2009 / Accepted: 17 December 2009 / Published online: 22 December 2009

(C) Springer Science+Business Media B.V. 2009

In this issue we present two speculative but exciting papers which may help provide insight into the nature of the dark energy of the Universe. The first of these by Hajdukovic (2010) is yet another exploration of the rich world of "cosmic coincidences" which are often described under the general heading of the Dirac large number hypothesis (Dirac 1938), but whose intellectual origins go back to Weyl a couple of decades earlier. Many such "coincidences" have now been investigated and discussed in the pages of this journal, most recently by Dinculescu (2009), Alfonso-Faus (2008) and Sorrell (2008). These attack the problem from a number of different viewpoints and working hypotheses, but involve similar expressions connecting fundamental atomic constants with cosmological quantities such as the Hubble Constant, the Black-Body temperature of the Universe and the Cosmological Constant. To quote Dinculescu (2009):

Is this a simple coincidence, or has (it) a deeper meaning? At this moment we simply do not know. Of course, we can invoke the anthropic principle (Barrow and Tipler 1986), or we can simply dismiss all these results, as precise as they are, as numerical coincidences, but they are too many, and too interconected.

In his paper Hajdukovic (2010) implicates pions as providing the link between the Hubble constant, $H$ and $\Omega_{\lambda}$, and suggests that:

It seems natural to me to suppose that the importance of pions, suggested by the preceding relations, is due

\section{Dopita ( $₫)$}

Research School of Astronomy \& Astrophysics, The Australian National University, Cotter Rd., Weston Creek, ACT 2611, Australia

e-mail: Michael.Dopita@anu.edu.au to "virtual" pions, which are, according to quantum field theory, an inherent part of vacuum fluctuations.

This potential link between quantum vacuum fluctuations and cosmological parameters is explored in a more rigorous way by Santos (2010) in the second of our two papers. He proposes that dark energy derives from the quantum vacuum fluctuations of all quantum fields in the Universe. Classical general relativity predicts that any stress-energy, $T_{\mu \nu}$, produces curvature of the space-time with a scaling parameter proportional to the Newton constant, $G$. However, if there is no classical stress-energy and in particular, if the vacuum expectation of the stress-energy tensor is zero, then there is no curvature to order $G$, but there will be an induced curvature to order $G^{2}$. Developing this idea further in his paper, Santos (2010) concludes:

the arguments of this paper strongly suggest that quantum vacuum fluctuations necessarily give rise to a curvature of space-time which roughly agrees with that attributed to dark energy. However a rigorous derivation would be required, which is not possible in the absence of a quantum gravity theory.

This is an idea of great elegance. Its development within the framework of a quantum gravity theory may not only elucidate the multiple "cosmic coincidences" problem, but it also provides a very simple explanation for the nature of dark energy without resort to more complex, ad-hoc, or exotic cosmological models. Dark energy emerges as a natural consequence of the quantum nature of the Universe in which we live. The challenge is now to develop a rigorous theory of quantum gravity.

\section{References}

Alfonso-Faus, A.: Astrophys. Space Sci. 318, 117 (2008) 
Dinculescu, A.: Astrophys. Space Sci. 323, 289 (2009)

Dirac, P.A.M.: Proc. R. Soc. A 165, 199 (1938)

Hajdukovic, D.S.: Astrophys. Space Sci. (2010), this volume. doi: 10.1007/s10509-009-0208-2
Santos, E.: Astrophys. Space Sci. (2010), this volume. doi:10.1007/ s10059-009-0204-6

Sorrell, W.H.: Astrophys. Space Sci. 317, 45 (2008) 